\title{
Building the Primary Healthcare Workforce for Interprofessional Collaboration
}

\author{
Nighat Huda ${ }^{1 *}$ \\ 'Department of Health Professions Education, Liaquat National Hospital \& Medical College, Karachi, Pakistan
}

In Primary Health Care settings, interprofessional collaboration has gained increased recognition to meet the changing needs of the growing elderly population, and those with several long-term chronic conditions. According to $\mathrm{WHO}$, interprofessional collaboration occurs when multiple healthcare workers from different professional backgrounds work together to provide comprehensive services by working with patients, their families, caregivers, and communities to deliver the highest quality of care across settings [1]. Evidence suggests that collaborative care delivered by a team of health care providers who have experienced interprofessional education (IPE) during their course of training provide cost-effective, person-centered care, and increase patients' satisfaction due to effective teamwork, and better communication among different health professionals [2]. Considering the primary health care services for the elderly population, a team of diverse health professionals providing well-coordinated care play a critical role in the elderly daily functioning and well-being. There is growing evidence that health care providers trained to work inter-professionally find themselves prepared to contribute as effective members of the collaborative primary health care team.

IPE and interprofessional collaborative care are interdependently linked with each other. IPE is when students from two or more professions learn about from and with each other to enable effective collaboration and improved health outcomes [3]. IPE role is significant in preparing a skilled workforce with competencies of team working skills, respect of each profession's scope of practice, and collaborative practice skills. Several international health organizations recommend countries to promote IPE by redesigning healthcare systems that advance interprofessional teamwork, enhance the quality of patient care, and improve health outcomes [4]. However, the greatest challenge in primary health care is the current practice of fragmented care delivered by multi-professionals who in most cases provide care independently, and exhibit ignorance and a negative attitude towards other health professionals' contributions. Despite resistance to moving towards interprofessional collaborative care, several developed

*Corresponding author: Nighat Huda, Department of Health Professions Education, Liaquat National Hospital \& Medical College, Karachi, Pakistan; Email: Nighat.Huda@lnh.edu.pk

Received: November 13, 2021; Revised: November 19, 2021; Accepted: November 22, 2021 DOI: https://doi.org/10.37184/lnjpc.2707-3521.3.26 and low and middle-income countries report successful implementation of collaborative care leading to improved communication and improved health outcomes.

In the context of Pakistan, both IPE and interprofessional collaborative care are of significant importance to strengthen primary care and improve population health outcomes. Pakistan is ranked as the sixth most populous country in the world while older adults are currently estimated to be 8 million and expected to rise to 27 million by $2050[5,6]$. Moreover, Pakistan's dismal performance in health is evident by its double-disease burden, rising fertility rate, and maternal and child mortality with widespread malnutrition among children and women. For concerned stakeholders, prioritization of $\mathrm{PHC}$ is critical to meet the vast majority of public diverse health needs across a lifetime. Similarly, Pakistan's health professional institutions will have to prepare health care providers for Pakistan's health system needs. Pakistan's current health professional education and training practices are mostly in silos and known to be embedded with the traditional mindset, culture and environment. A strong barrier to IPE in Pakistan is associated with the hierarchical organisation of health care with medical doctors as the central providers with optimal structural support focus on medical sciences and medical education while nurses and other health professionals face immense challenges with regard to recognition and acceptance as prestigious members of the health care team. Similar challenges such as leadership, resources, stereotypes and attitudes, and accreditation have been discussed in the literature. Pakistan can benefit from the experiences of health professional institutions in developed countries that have established their own successful IP collaborative models for improved cost-effective health outcomes and quality patient care. Nevertheless, the opportunity for sustainable IPE programs is greater if supported by established accreditation bodies of medicine, nursing and pharmacy. Globally, there are even examples of an independent accreditation body for interprofessional education and collaborative practice. The ongoing COVID-19 pandemic should be a wakeup call for stakeholders of Pakistan to realize that strong primary health care is the pillar of strong health systems, and the presence of a collaborative practice work force even in an epidemic situation will ensure the continuation of the right care, at the right time, and at a much lesser cost. 


\section{REFERENCES}

1. WHO. Framework for action on interprofessional education \& collaborative practice. Geneva: World Health Organization 2010. Available at:http://whqlibdoc.who.int/hq/2010/WHO_HRH_ HPN_10.3_eng.pdf(Accessed on: Nov 11, 2021).

2. Frenk J, ChenL, Bhutta ZA, Cohen J, Crisp N, Evans T, et al. Health professional for a new health century: transforming education to strengthen health systems in an independent world. Lancet 2010; 376(9756): 1923-58.

3. Miller R, Scherpbier N, van Amsterdam L, Guedes V, Pype P. Interprofessional education and primary care: EFPC position paper. Prim Health Care Res Dev 2019; 20: e138.
4. van Weel C, Kassai R, Qidwai W, Kumar R, Bala K, Gupta PP, et al. Primary healthcare policy implementation in South Asia. BMJ Glob Health 2016; 1: e000057.

5. Primary Health Care Systems (PRIMASYS) Comprehensive case study from Pakistan. World Health Organization 2017. https:// www.who.int/alliance-hpsr/projects/AHPSR-PRIMASYS-Pakistancomprehensive-v2.pdf

6. Cassum LA, Cash K, Qidwai W, Vertejee S. Exploring the experiences of the older adults who are brought to live in shelter homes in Karachi, Pakistan: a qualitative study. BMC Geriatr 2020; 20(1): 8 . 